\title{
Bibliotecas integradas: alfabetización en información como estímulo de cooperación
}

\author{
Por Miguel-Ángel Marzal y Pablo Parra-Valero
}

Resumen: Se presentan las bibliotecas integradas como modelo idóneo de cooperación en su dimensión educativa. Su carácter "integrado" las convierte en plataforma de convergencia para bibliotecas con función educativa, por administrar y gestionar cooperativamente recursos y servicios de información, contenidos y recursos humanos. Se analizan los principales modelos de bibliotecas integradas, con especial atención a la alfabetización en información, para llegar a analizar el caso australiano por su liderazgo y su aportación a la alfabetización en información. Se analizan los progresos en España, sus experiencias, desde la perspectiva de un análisis DAFO, para destacar las oportunidades que ofrecen. Se propone un modelo de programa de alfabetización en información para bibliotecas integradas, elaborado por los autores para Baratz, en el que se diseña un programa educativo competencial, conforme al desarrollo de capacidades lectoescritoras en el ámbito digital y de la web 2.0.

Palabras clave: Bibliotecas integradas, Alfabetización en información, Alfin, Cooperación bibliotecaria, Educación para la sociedad del conocimiento, E-learning.

Title: Joint-use libraries: information literacy as a stimulus for cooperation

Abstract: This article presents the educational role of jointuse libraries as a suitable model of cooperation. Its integrated character allows it to become a platform of convergence for libraries with an educational function in order to effectively administer and manage information services, contents and li-

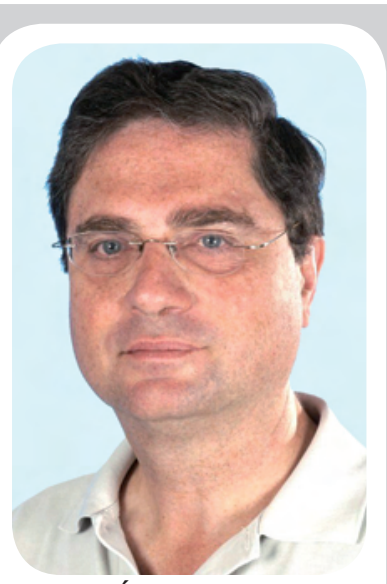

Miguel-Ángel Marzal, doctor y premio extraordinario en historia por la Univ. Autónoma de Madrid, es profesor titular de biblioteconomía y documentación en la Univ. Carlos III de Madrid. Miembro de comités científicos de congresos nacionales e internacionales, $y$ autor de varias monografías y artículos en revistas. Dirige el equipo de investigación Doteine, integrado como co-director en el equipo de investigación Acrópolis, y es miembro del Instituto de Investigación Agustín Millares de la Univ. Carlos III.

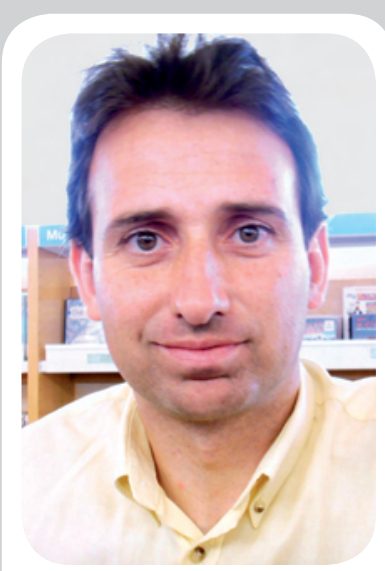

Pablo Parra-Valero es licenciado en ciencias de la información, especialidad periodismo, por la Universidad Complutense de Madrid y diplomado en biblioteconomía y documentación en la Universidad Carlos III de Madrid (UC3M). Es doctorando del Departamento de Biblioteconomía y Documentación de la Universidad Carlos III de Madrid, colaborador en los proyectos del equipo de investigación Acrópolis, y desde el año 2002 responsable de la Biblioteca Municipal de Morata de Tajuña (Madrid)

brary human resources. Considering the principal models of joint-use libraries, we pay special attention to the Australian case for its leadership in the topic and its contribution to information literacy. Experience, progress and, even more, the opportunities that the model offers in Spain are analyzed through a SWOT matrix. Finally, we propose a model programme on information literacy for joint-use libraries, developed by the authors for Baratz Servicios de Teledocumentación S. A., that provides for the development of reading and writing skills in the digital area and in the world of Web 2.0.

Keywords: Joint-use libraries, Information literacy, Library cooperation, Education for Knowledge Society, E-learning.

Marzal, Miguel-Ángel; Parra-Valero, Pablo. "Bibliotecas integradas: alfabetización en información como estímulo de cooperación”. El profesional de la información, 2010, septiembre-octubre, v. 19, n. 5, pp. 463-468.

DOI: $10.3145 /$ epi.2010.sep.03

\section{Bibliotecas integradas}

Se trata de un modelo cooperativo de biblioteca que nace y se planifica con más de una función, de forma que puedan suministrarse servicios bibliotecarios diferentes dentro de unas mismas instalaciones. En inglés se conocen por joint-use libraries. La integración da lugar a un modelo cooperativo bibliotecario sumamente eficiente para la administración de los recursos técnicos y humanos, la gestión de servicios informativos, el uso de los espacios y el desarrollo de colecciones documentales y contenidos web.

El modelo ha evolucionado en los últimos veinte años. En España el grupo de trabajo Sistemas Urbanos de Bibliotecas, surgido en las Jornadas de cooperación bibliotecaria 2000, elaboró un documento en el que recogieron formas complejas de cooperación en bibliotecas de una misma ciudad.

http://travesia.mcu.es/portalnb/jspui/handle/10421/389 
http://www.mcu.es/bibliotecas/CE/Cooperacion/Cong Jornadas/Jornadas/Logrono2000.html

La cooperación siempre ha estado presente en la filosofía de trabajo de las bibliotecas. En algunos casos fue la biblioteca pública la que estableció unidireccionalmente vínculos de colaboración con las instituciones educativas, asumiendo una mayor responsabilidad en su función formativa y ofreciendo espacios educativos. En aquellos lugares donde no existía una biblioteca pública eran las escuelas las únicas que podían ofrecer el servicio bibliotecario a la comunidad.

Las bibliotecas integradas surgen de la formalización de acuerdos entre dos o más autoridades diferentes que proporcionan el acceso a servicios e instalaciones a dos o más grupos de usuarios en igualdad de condiciones (Bundy, 2003). Allá donde la red bibliotecaria ha sido más frágil, o donde las escolares han tenido por múltiples razones un escaso desarrollo, la integración ha permitido su notable mejora.

Existen diversos formatos de integración, pero todos tienen como denominador común la optimización de espacios y servicios. Sin embargo en años recientes las necesidades de alfabetización en información (alfin) han hecho que este tipo cooperativo adquiera nuevas dimensiones.

\section{"La integración permite aumentar la eficiencia, hacer un uso más eficaz de los espacios, y mejorar las colecciones documentales y los contenidos web"}

\section{Modelos de biblioteca integrada. Estímulo de la alfin}

Existen ejemplos de bibliotecas integradas en distintos países que se agrupan en tres grandes bloques: norteamericano, europeo y australiano (Marzal; Parra-Valero, 2009). El modelo apuntado en los países nórdicos europeos, en coincidencia con Australia, presenta la peculiaridad de la relevancia que se otorga a la implementación de programas de alfin.

La decidida apuesta de los países nórdicos europeos por una educación de calidad les ha llevado a ostentar reiteradamente los primeros puestos en el informe Pisa (Programme for International Student Assessment), que es una radiografía de la salud y calidad del sistema educativo. Por su parte, Australia y Nueva Zelanda implementaron un modelo bibliotecario instalado en el sistema educativo, lo que les llevó a protagonizar el proceso normativo de las competencias informacionales. Los programas de alfin se convirtieron así en una oportunidad para una reinterpretación y un salto cualitativo importante en el modelo cooperativo de bibliotecas integradas. La necesidad de obviar la brecha digital hizo consolidar la cooperación.

http://www.pisa.oecd.org/

\section{"Los programas de alfin son una oportunidad para reinterpretar y dar un salto cualitativo en el modelo cooperativo de bibliotecas"}

\section{Modelo de South Australia}

Que sepamos, la región de Australia del Sur es el único lugar del mundo en el cual se ha adoptado la política oficial de instalar bibliotecas públicas dentro de los centros educativos. El excesivo retraso en poder contar con bibliotecas públicas independientes -fue el último estado australiano en conseguirlo-, con el paso del tiempo se ha convertido en ventaja, pues conjuntando bibliotecas escolares y públicas sacan mejor rendimiento a la financiación gubernamental. La primera biblioteca en abrir sus puertas fue la de Pinnaroo en 1977. Su temprano éxito ayudó a persuadir a otras poblaciones, y a comienzos de los noventa se contabilizaban casi 50 bibliotecas integradas. En la actualidad, según la edición de 2010 del Directory of Australian public libraries, hay 92 bibliotecas integradas en Australia, de las cuales el 62\% (57) se encuentra en South Australia.

\section{http://lists.webjunction.org/libweb/Aus_Australia.html}

Si bien es cierto que actualmente se inauguran bibliotecas integradas concebidas así desde su origen, en la mayoría de los ejemplos encontrados en South Australia el servicio de biblioteca pública fue incorporado a una biblioteca educativa ya existente, por lo que es probable que las bibliotecas joint-use jamás hubieran aparecido de no ser por la existencia de una sólida infraestructura de bibliotecas escolares. Su exitosa longevidad se debe a la existencia del School / Community Library Committee, con su rigurosa planificación e investigación, que informa, resuelve las dificultades surgidas y revisa los acuerdos establecidos (Bundy, 1997). Junto a éste destacan otros factores como la proliferación de recomendaciones, informes y estudios positivos elaborados durante los últimos 40 años, entre los que figuran las tesis de Brown (1986) y Bundy (1997). 


\section{Análisis DAFO de la aplicación del modelo en España}

Desde la idea de las Misiones Pedagógicas, al frente de las cuales estaba María Moliner con su Plan nacional de bibliotecas, considerado como el primer testimonio español, se repiten dos rasgos que caracterizan la baja aplicación del modelo joint-use:

- el tradicional déficit que arrastran las bibliotecas escolares; y

- la constatación de experiencias no formalizadas de bibliotecas integradas.

Tampoco ayudó el hecho de que las pocas experiencias llevadas a cabo no fueran satisfactorias por falta de continuidad o de apoyo institucional.

En la última década han surgido nuevos proyectos de biblioteca integrada con buenos resultados, lo cual ha animado a otros municipios a emular esta experiencia cooperativa. Existen 35 bibliotecas escolares abiertas al público en general, sin embargo, su nivel de integración es mínimo y, por lo general, biblioteca pública y biblioteca escolar actúan como meros huéspedes ya que comparten un edificio pero funcionan separadamente con su propio horario de apertura. El matrimonio efectivo entre bibliotecas tiene lugar cuando la instalación es pública y escolar de forma simultánea sin restricción alguna (McNicol, 2008).

Con el objeto de analizar las posibilidades de desarrollo y la problemática real del modelo en España, se ha realizado un análisis DAFO (debilidades, amenazas, fortalezas y oportunidades) de las bibliotecas integradas.

\section{"Un factor crítico de éxito es el perfil profesional de la persona encargada de gestionar las bibliotecas integradas"}

Una de las principales debilidades que dificultan la difusión y el desarrollo de bibliotecas integradas es que, salvo en Galicia, Cantabria y Baleares, no hay alusiones específicas en las leyes bibliotecarias autonómicas que permitan su creación. También se echa en falta la existencia de documentos normativos, estándares, pautas y recomendaciones que puedan ser útiles a la hora de plantear un proyecto.

Otra debilidad fundamental y factor crítico de éxito, es el perfil profesional que debe reunir la persona encargada de gestionar este tipo de bibliotecas. El problema se resolvió en Australia con la figura del teacher librarian, poseedor de una doble cualificación como bibliotecario y maestro, y cuya misión no es organizar y ordenar la biblioteca sino prestar apoyo al resto del profesorado para elaborar el currículo escolar de los estudiantes y la alfin.

Además encontramos otras debilidades como la falta de consenso terminológico para referirse a esta fórmula de cooperación (Parra-Valero, 2008), la inexistencia de convenios que regulen esta combinación de bibliotecas y que aseguren su permanencia en el tiempo más allá de la voluntad de las autoridades municipales o los directores de los centros de enseñanza, y otras de carácter más funcional como la ausencia en algunos casos de una doble entrada que permita el acceso de los alumnos desde las aulas, y la del público desde el exterior.

A todo ello se une el recelo con que la integración bibliotecaria se ha visto hasta ahora y el resquemor a que desaparezca la biblioteca escolar. Sin embargo la propia filosofía del joint-use indica que su materialización nunca debe suponer la desaparición o la negación de un tipo concreto de biblioteca ya que cada una tiene unos objetivos y una misión irreemplazable.

Frente a estas debilidades y amenazas encontramos fortalezas como algunos ejemplos que pueden guiar la implantación de esta forma de cooperación. Uno de ellos es el programa Bibliotecas de barrio de Collado Villalba (Madrid) que este año ha celebrado su décimo aniversario con la inauguración de su novena biblioteca integrada, lo que le convierte en el municipio con mayor número de instalaciones.

Otro caso a resaltar es el proyecto de Bibliotecas de doble uso de Castilla-La Mancha iniciado en 2007 y que se basa en unos acuerdos a tres bandas que comprometen a las consejerías de Cultura y Educación de Castilla-La Mancha y a los cinco ayuntamientos participantes en este momento, cuyas bibliotecas están conectadas al catálogo colectivo de la Red de Bibliotecas de Castilla-La Mancha. El proyecto ha planteado unas condiciones mínimas que deben caracterizar a estas bibliotecas en cuanto a instalaciones, personal, colección y servicios y, además, cuenta con unas Directrices para bibliotecas públicas de doble uso en fase de borrador.

Por último, se encuentran las bibliotecas integradas de Lorca (Murcia) incluidas dentro de la Red de Bibliotecas Municipales de esta localidad. Se trata de un proyecto municipal y no regional, por lo que el consistorio aporta el personal especializado, el mobiliario, la adquisición de fondos más generales y el tratamiento técnico de los mismos. Los ejemplos de Lorca constituyan un modelo a tener en consideración por múltiples razones como: el planeamiento de las instalaciones, su integración y apertura a la comunidad (en lo que ha influido la colocación de grandes rótulos luminosos con 
la denominación "biblioteca pública" en la fachada de los centros educativos), la proyección de futuro, y hasta por la propia disposición orográfica y la división del término municipal en 39 pedanías.

Entre las principales oportunidades del modelo de biblioteca integrada destaca, en general, su posición privilegiada para combatir la exclusión social y los efectos de la brecha digital.

\section{Programas de alfin para bibliotecas}

El panorama descrito confirma en el caso español la oportunidad que para el modelo cooperativo de bibliotecas integradas supone contar con programas de alfabetización en información, como pilares en su diseño y desarrollo. Éste fue el estímulo que llevó a los autores de este trabajo a proponer a la empresa Baratz un proyecto de investigación aplicada consistente en la elaboración de un programa de alfin para su difusión y uso en bibliotecas integradas.

El proyecto se planteó como una de las conclusiones del $6^{\circ}$ congreso Desarrollo de habilidades informativas (DHI) organizado por el Servicio de Bibliotecas de la Universidad Autónoma de Ciudad Juárez en 2008.

\section{http://bivir.uacj.mx/dhi/6e/default.asp}

$\mathrm{Su}$ arquitectura se componía de los siguientes elementos: descubrimiento, presentación, inspección, recomendación, combinación y reflexión.

En distintas universidades españolas se ha iniciado la enseñanza de materias transversales, como las habilidades informacionales. La implicación en ello de las bibliotecas universitarias ha traído consigo que el resto de bibliotecas también se involucre, tanto en acciones de educación formal como no formal, y lógicamente las bibliotecas integradas las que más.

Como en general a los bibliotecarios no les había recaído impartir docencia, la disponibilidad ahora de un programa de alfin resulta muy útil.

La metodología utilizada para el diseño curricular de esta "materia" se basa en:

- adecuación a los principios del modelo DeSeCo (Definición y Selección de Competencias Básicas);

- formación continua y aprendizaje permanente;

- tipo de e-learning que no es para una simple actualización de contenidos o el dominio de herramientas, sino que permite una verdadera asimilación de conocimientos;

- blended learning (modo de aprendizaje que combina la enseñanza presencial y la online). Se usa una plataforma digital pero luego se hace un seguimiento de las materias en clases presenciales;
- formulación de objetivos según las Normas de alfabetización en información editadas por Anziil (Australian and New Zealand Institute for Information) en 2004.

El objetivo no era disponer de un simple tutorial sino de un programa de alfin completo.

La preferencia por las Normas Anziil obedecía a dos motivos: su filosofía y diseño desde Australia, donde el modelo de bibliotecas integradas está muy maduro; y por la figura de Alan Bundy, bibliotecario y científico muy implicado en la elaboración de las normas y en el desarrollo de las bibliotecas integradas. En marzo de 2010 Bundy hizo una estancia de investigación en la Universidad Carlos III de Madrid, participando en actividades del proyecto con Baratz.

El proyecto contemplaba la formulación teórica ya descrita, la programación en siete módulos educativos y su edición para web. Un aspecto que se cuidó especialmente fue la inclusión de la evaluación en el programa, uno de los temas fundamentales y más atractivos en la investigación de alfabetización en información. Para ello se elaboró un estado del arte buscando la literatura científica existente sobre indicadores y modelos de evaluación de alfin (particularmente la tesis de Peña-López), así como y esencialmente, modelos de indicadores competenciales cuantitativos y cualitativos. De éstos, se han tenido especialmente en cuenta los impulsados por la Comisión Europea a través del Digital Literacy High-Level Expert Group y el presentado en Sibis (Statistical Indicators Benchmarking the Information Society), así como los diseñados por el Unesco Institute for Statistics, especialmente a partir de la Guide to measuring information and communication technologies (ICT) in education, 2009.

El proyecto se inició en octubre de 2009 y está prevista su edición digital en septiembre de 2010.

http://ec.europa.eu/information_society/eeuropel i2010/digital_literacy/index_en.htm

http://www.sibis-eu.org/

http://www.unescobkk.org/fileadmin/user_upload/ aims/Publications/ICT_Guide_EN.pdf

Salvo los Módulos 0 y 6, el resto tienen una misma estructura, que contempla seis elementos instructivos:

- guía de uso, donde se plantean las competencias a conseguir, así como todos los principios teóricos necesarios para entender y asimilar el módulo;

- ejemplos ilustrativos, diseñados para ser utilizados junto a la guía de uso, con el fin que los principios teóricos, sean más eficazmente asumidos y transformados en habilidades prácticas por parte del alumno;

- software y recursos digitales; 
- material de apoyo para ayudar a que el alumno comprenda la utilidad de la aplicación;

- práctica del módulo, cuya correcta ejecución por el alumno acreditará la consecución de las competencias;

- tests de autoevaluación para que el alumno haga un seguimiento de su progreso.

El Módulo 0 inicia el programa con un cuestionario sobre uno o varios recursos educativos en web para medir el nivel de alfin del alumno al comenzar.

El Módulo 6 es un modelo de indicadores para uso del personal docente-bibliotecario, que permite supervisar el proceso y presentar informes a las autoridades académicas y administrativas pertinentes sobre los progresos de los alumnos, así como de la calidad percibida del programa de alfin.

\section{"Las bibliotecas integradas crean economías de escala"}

Los Módulos 1 a 5 se diseñaron conforme a las Normas Anziil, así como a los principios competenciales que en su día publicara Bruce (2003). Sólo el Módulo 1 coincide con los contenidos de la formación de usuarios en línea, para a partir de entonces acogerse a la concepción de la alfin recogida al final del punto 2 , en una senda hacia la alfabetización en información 2.0. De este modo, los contenidos que se programan son:

- Módulo 1 (normas 1 y 2 de Anziil), para diseño de la búsqueda y estrategias de recuperación de información, junto con el manejo experto, selección y evaluación de las fuentes de información. Se sigue por su gran utilidad el modelo Gavilán (definir, buscar, analizar y sintetizar):

\section{http://www.eduteka.org/pdfdir/ModeloGavilan.php}

- Módulo 2 (norma 3). Uso experto de instrumentos de organización y gestión de contenidos (tesauros visuales y asociativos, topic maps, ontologías).

- Módulo 3 (norma 4). Consecución de una lectura digital correcta usando mapas conceptuales propios del alumno.

- Módulo 4 (norma 5). Conocimiento y empleo hábil y educativo de las redes sociales y la web 2.0, como elementos fundamentales en el logro de una cultura digital comprensiva.

- Módulo 5 (norma 5). Escritura digital -el otro elemento de la alfabetización en información-, y edi- ción ética de los contenidos en objetos de aprendizaje propios de cada alumno.

La voluntad de que el programa no sea sólo un tutorial ha hecho que el equipo de informáticos de Baratz haya insistido en la mejora de los aspectos interactivos y visuales.

\section{Conclusiones}

Las bibliotecas integradas crean economías de escala, permitiendo tener servicios bibliotecarios y gestionar contenidos documentales de forma más eficaz, algo que es especialmente interesante en épocas de crisis.

La reunión de recursos y servicios documentales, informativos, bibliotecarios, informáticos, audiovisuales, pedagógicos..., convierte a las bibliotecas integradas en ideales para su transformación en centros de recursos, a modo de los crais en las universidades.

La web añade oportunidades a este tipo de bibliotecas, pues son apropiadas para ejercer como centros de alfin, uno de los factores más decisivos en el progreso hacia la sociedad del conocimiento.

Esta circunstancia es la que hace más perentoria la necesidad de diseñar y editar programas de alfin adaptados no sólo a las normas y estándares publicados, sino sobre todo a cada comunidad de usuarios.

Se hace preciso pasar de las formulaciones teóricas hacia propuestas concretas que permitan a los bibliotecarios con responsabilidades formativas disponer de aplicaciones para desempeñar con eficacia la acción en alfabetización en información. Éste es el objetivo del proyecto aquí descrito, que ha implementado Baratz.

\section{Bibliografía}

Amey, Larry J. Combining libraries: the Canadian and Australian experience. Metuchen, NJ: Scarecrow Press, 1987.

Brown, Anthony J. Joint-use college and community libraries: a comparative study, with special reference to developments in South Australia, 19781984. Adelaide: South Australian Institute of Technology, 1986.

Bruce, Christine-Susan. "Las siete caras de la alfabetización en información en la enseñanza superior". Anales de documentación, 2003, n. 6, pp. 289-294.

Bundy, Alan. Widened horizons: the rural school community libraries of South Australia. Adelaide: Auslib Press, 1997.

Bundy, Alan. "Joint-use libraries: the ultimate form of cooperation". En: McCabe, Gerard; Kennedy, James. Planning the modern public library building. Westport: Libraries Unlimited, 2003, pp. 129-148.

Marzal, Miguel-Ángel. "La alfin como innovación educativa: objetivos, diseño curricular, impacto evaluativo y espacio para un modelo educativo competencial". En: $6^{\circ}$ Desarrollo de habilidades informativas (DHI). Ciudad Juárez, oct. 2008

http://bivir.uacj.mx/dhi/6e/presentaciones/MiguelAngelMarzal.mht

Marzal, Miguel-Ángel. "Evaluation of information literacy programmes in higher education: strategies and tools". Information and digital competencies in higher education [online monograph]. Revista de universidad y sociedad del conocimiento (Rusc), 2010, July, v. 7, n. 2, pp. 26-35. 
http://rusc.uoc.edu/ojs/index.php/rusc/article/view/v7n2-marzal/v7n2marzal-eng

Marzal, Miguel-Ángel; Parra-Valero, Pablo. "La cooperación de la biblioteca escolar y la pública: alfabetización en información, biblioteca integrada y el mundo virtual". Revista general de información y documentación, 2009, v. 19, n. 1, pp. 212-241.

http://www.ucm.es/BUCM/revistas/byd/11321873/articulos/RGID 0909110221A.PDF

McNicol, Sarah. Joint-use libraries: libraries for the future. Oxford: Chandos, 2008.

Parra-Valero, Pablo. "La biblioteca integrada: un modelo de cooperación entre bibliotecas públicas y otras unidades de información”. En: Actas del IV Congreso Nacional de Bibliotecas Públicas, 2008, pp. 193-203.

Peña-López, Ismael. Measuring digital development for policy-making. models, stages, characteristics and causes.

http://ictlogy.net/articles/20090908_ismael_pena-lopez_-_measuring_ digital_development_for_policy-making.pdf

Queensland Government. Guidelines for the development of joint-use school-community libraries, Queensland, 1996. http://education.qld.gov.au/library/docs/joint-use.pdf

Unesco Institute for Statistics. Guide to measuring information and communication technologies (ICT) in education, 2009, 139 pp

http://www.unescobkk.org/fileadmin/user_upload/aims/Publications/ICT_ Guide_EN.pdf

Miguel-Ángel Marzal. Departamento de Biblioteconomía y Documentación, Universidad Carlos III de Madrid. c/ Madrid, 128. 28903 Getafe, Madrid.

mmarzal@bib.uc3m.es

Pablo Parra-Valero. Ayuntamiento de Morata de Tajuña, Biblioteca Municipal. Tarayuela, 1. 28530 Morata de Tajuña, Madrid.

biblioteca@ayuntamientodemorata.com

\section{Revista científica y profesional en español líder en el área de información, bibliotecas y nuevas tecnologías de la información.}

El profesional de la - información-

Nov 2010 Medios de comunicación en intemet Enero 2011 Psicología y sociología de la información Marzo 2011 Archivos administrativos e intranets Mayo 2011 Información de las administraciones públicas Julio 2011 Fotografía y bancos de imágenes

Sept 2011 Inteligencia competitiva

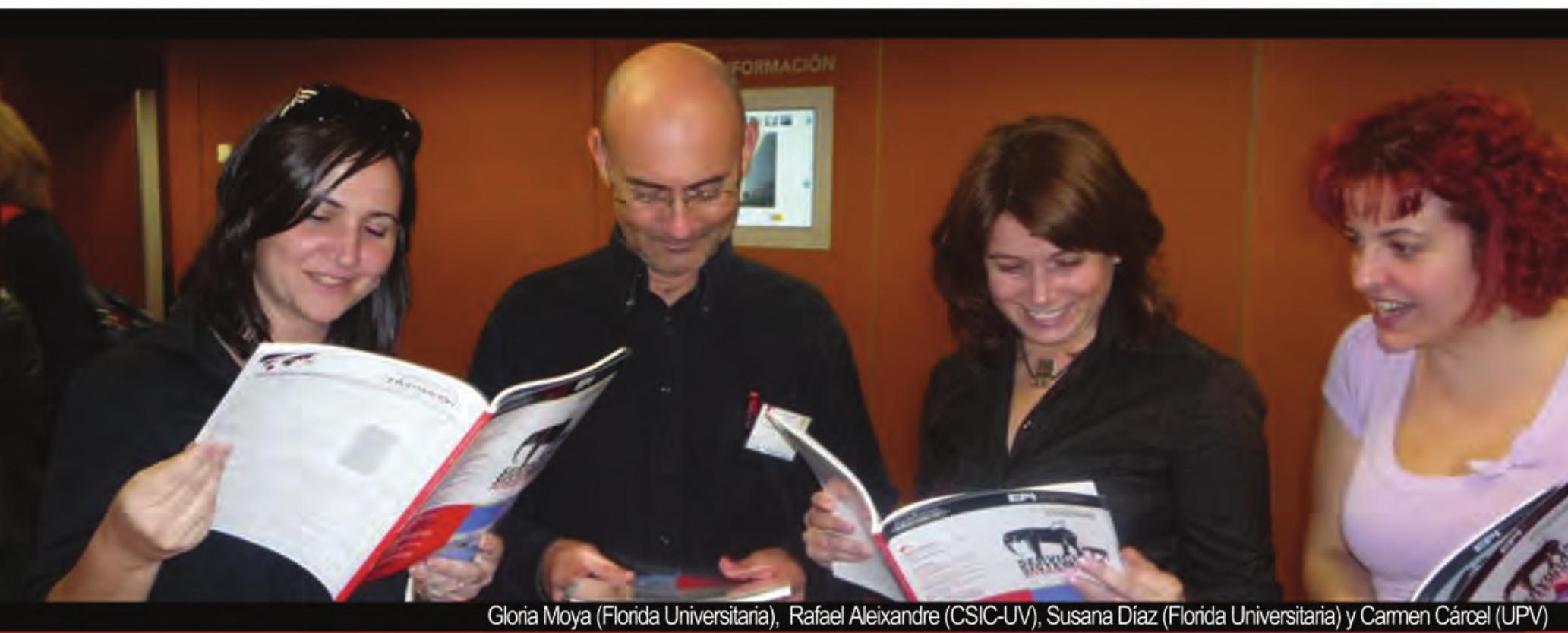

La 1a indexada por Science Citation Index

(Thomson ISI), y Scopus (Elsevier), además de otras 27 bases de datos y servicios bibliográficos.
Textos completos

(1992-2008) en OPEN ACCESS )

disponibles desde nuestra web.

\section{www.elprofesionaldelainformacion.com}

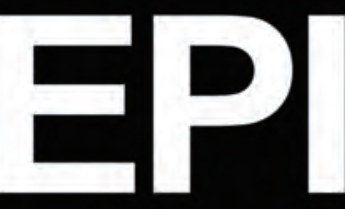

REDACCION: epi@elprofesionaldelainformacion.com

SUSCRIPCIONES: suscripciones@elprofesionaldelainformacion.com

PUBLICIDAD: publici@elprofesionaldelainformacion.com

Apartado 32.280, 08080, Barcelona Tel. +34-609 352954 